\title{
Nuevos retos y perspectivas de investigación en Educación Física New challenges and research perspectives in Physical Education
}

$\mathrm{E}$ I presente monográfico "Nuevos retos y perspectivas de investigación en Educación Física" es una iniciativa que surge desde la Red internacional de investigación en Educación Física y Promoción de Hábitos Saludables (www.edufisaludable.com). Esta red, financiada por el Consejo Superior de Deportes en sus convocatorias de 2019 y 2020, reúne en la actualidad a más de 50 investigadores de todo el panorama nacional e internacional. En el contexto español, la investigación en el ámbito de la Educación Física se ha incrementado en los últimos años, no solo desde una perspectiva cuantitativa, sino también cualitativa, lo que muestra la fortaleza y el buen estado de forma de la Educación Física en nuestro país. Sin embargo, se estima necesario seguir investigando, atendiendo al sistema educativo actual, en qué medida desde la asignatura de Educación Física se puede promover la adquisición de hábitos saludables por parte de los escolares. Por ello, desde esta Red de investigación se ha pretendido dar un impulso en la visibilidad de la investigación sobre esta unión entre la Educación Física y la promoción de hábitos saludables.

A pesar de los beneficios físicos, psicológicos, sociales y cognitivos que reporta la adopción de un estilo de vida saludable, la mayoría de los niños y adolescentes no cumple las recomendaciones de actividad física (AF) y de otros comportamientos relacionados con la salud (e.g., duración de sueño, tiempo de pantalla, patrones de alimentación, etc.) (Marques et al., 2018). Por ejemplo, en lo que a la AF se refiere, un estudio reciente llevado a cabo en 146 países y que incluía 1.6 millones de adolescentes mostró que el $81 \%$ de los participantes entre 11 y 17 años no cumplía las recomendaciones internacionales (Guthold et al., 2019). A nivel nacional, diferentes estudios realizados recientemente, entre los que podemos destacar el estudio PASOS de la Fundación Gasol (Gasol Foundation, 2019), el informe "Mujer Joven y Actividad Física" de la Fundación Mapfre (Luque \& Del Villar, 2019) o el estudio HBSC 2018 (Moreno et al., 2019), también alertan de la insuficiente práctica de actividad física de niños y adolescentes, lo que unido a un menor cumplimiento de las recomendaciones de otros comportamientos de riesgo (e.g., un alto tiempo de pantalla), la prevalencia de sobrepeso y obesidad se ha incrementado en los últimos años entre la población joven.

Una gran cantidad de estudios señalan que la adopción de un estilo de vida saludable podría prevenir o reducir la mortalidad o morbilidad provocada por las enfermedades no transmisibles (ENT; e.g., enfermedades cardiovasculares, diabetes, etc.) (Gakidou et al., 2017). Para combatir este grave problema de salud pública, la Organización Mundial de la Salud (OMS) ha elaborado diferentes planes como el "Plan de acción mundial para la prevención y control de las ENT 2013-2020" o el "Plan de acción mundial sobre actividad física 2018-2030: Más personas activas para un mundo más sano". Los objetivos de este último plan pretende aumentar los niveles de AF en adolescentes y adultos en un 15\% en 2030 a través de cuatro objetivos estratégicos: 1) crear sociedades activas; 2) crear entornos activos; 3) crear personas activas; y 4) crear sistemas activos. A pesar de que se han invertido gran cantidad de recursos humanos y económicos, diferentes revisiones sistemáticas han mostrado la falta de efectividad, sostenibilidad y trasferencia de las intervenciones realizadas sobre promoción de hábitos saludables (Love et al., 2019). Dentro de todos los contextos desde los que pueden implementarse este tipo de programas, el entorno escolar parece ampliamente reconocido como un ambiente privilegiado debido a que: 1) reúne a toda la población durante una larga etapa de su vida; 2) la etapa infantil y adolescente es crítica en la transferencia de hábitos saludables a la etapa adulta; 3) permite atender, de forma inclusiva, a grupos prioritarios hacia la práctica de AF, como las chicas, el alumnado con un menor estatus socio-económico o con algún tipo de diversidad funcional; y 4) permite servir de eslabón para involucrar a todos los agentes de la comunidad educativa y social e implementar estrategias a través de la vía curricular y no curricular.

Sin embargo, una recopilación de estudios previos también ha mostrado una falta de eficacia de los programas de intervención escolares destinados a la mejora de la práctica de AF y otros hábitos saludables en niños y adolescentes (Borde et al., 2017). En la misma línea, otros estudios han revelado que las pequeñas mejoras provocadas por los programas escolares disminuyen o se diluyen 
con el tiempo, lo que sugiere que dichos programas no resultan tampoco eficaces a largo plazo (Nguyen et at., 2016). Por tanto, es necesario identificar las causas que pueden justificar la falta de eficacia de los programas escolares (Cassar et al., 2019). Recientemente, Sallis (2018) ha identificado las intervenciones multinivel, a partir de modelos ecológicos, como una posible solución para atender desde los programas escolares los diferentes factores de influencia de los comportamientos saludables. Sin embargo, todavía existe un número muy limitado de estudios que hayan implementado este tipo de intervenciones a nivel escolar.

Por otro lado, de todos los agentes del ámbito educativo, algunos autores han destacado al profesor de Educación Física como uno de los agentes educativos más influyentes en la práctica de AF, así como otros hábitos saludables, en el alumnado (Slingerland \& Borghouts, 2011). El profesorado de Educación Física puede influir tanto de forma directa (e.g., contribuyendo en las clases de Educación Física al cumplimiento de los niveles de AF moderada y vigorosa [AFMV]) como de forma indirecta (e.g., transmitiendo conocimientos, actitudes y competencias necesarias para que el alumnado adopte un estilo de vida saludable) en la promoción de hábitos saludables (Slingerland \& Borghouts, 2011). Debido al reducido número de horas en esta asignatura (i.e., en España cuenta tan solo con una carga lectiva de dos horas semanales) y a que determinados estudios han concluido que los niveles de AFMV en las clases de Educación Física solo suponen el $36 \%$ del tiempo total de las sesiones (Hollis et al., 2017), parece especialmente relevante dicha vía de promoción indirecta. Consecuentemente, una de las prioridades del profesorado de Educación Física es proporcionar a los escolares los conocimientos, habilidades y actitudes necesarias que garanticen una práctica de actividad física en el tiempo de ocio (Hagger \& Chatzisarantis, 2012; Taylor et al., 2009; Whitehead, 2010).

Las experiencias acumuladas en Educación Física son determinantes a la hora de adoptar o no un estilo de vida saludable. Las experiencias negativas acumuladas de forma reiterada se asocian a sentimientos de incompetencia y al abandono de la práctica de AF (Beltrán-Carrillo et al., 2019). Sin embargo, experiencias positivas en las clases de Educación Física en la infancia se han visto relacionadas con una mayor intención de realizar AF y con la reducción del comportamiento sedentario en la etapa adulta (Ladwig et al., 2018). En este sentido, el profesorado de Educación Física tiene una responsabilidad directa en las experiencias generadas en sus clases. El análisis del estilo interpersonal docente del pro- fesorado de Educación Física debido a su repercusión en los procesos motivacionales y consecuencias generadas en el alumnado (e.g., práctica de AF) parece, por tanto, de suma importancia (Vasconcellos et al., 2020). En este sentido, son necesarios más estudios que examinen la influencia de los elementos pedagógicos del docente de Educación Física (e.g., aplicación de modelos pedagógicos, el uso de estilos motivacionales en la adquisición de experiencias positivas en las clases de Educación Física, etc.) y, consecuentemente, la influencia del docente en la práctica de AF presente y futura de los jóvenes y adolescentes.

Todos estos estudios justifican y argumentan la necesidad de seguir profundizando en la investigación relacionada con la Educación Física y su repercusión sobre el desarrollo de hábitos saludables, tanto de forma directa como de forma indirecta. En este monográfico se abordan un total de 11 estudios que esperamos puedan aportar luz y avanzar en el conocimiento científico de esta línea de investigación. Dentro de las distintas temáticas abordadas en la Red internacional de Investigación Educación Física y Promoción de Hábitos Saludables se han destacado tres líneas prioritarias de trabajo que pueden guiar el desarrollo de investigaciones sobre esta temática en la actualidad. Estas tres líneas son: 1) el uso de modelos pedagógicos en Educación Física; 2) el estudio de la intervención docente y sus consecuencias sobre distintas variables motivacionales en Educación Física; y 3) desarrollo de intervenciones desde los centros escolares y desde la Educación Física para la promoción de hábitos saludables. Estas tres líneas de trabajo reflejan las posibilidades de generar efectos positivos en los niños y adolescentes desde la Educación Física y contribuir a través de experiencias positivas en la AF. Estas líneas de trabajo también se han visto reflejadas en este monográfico.

Dentro de la línea de Modelos Pedagógicos encontramos el estudio "Enseñanza y aplicación de los test de condición física en Educación Secundaria: Una propuesta basada en el Modelo de Educación Deportiva", desarrollado por Carriedo et al. (2020), que aborda un contenido complejo pero implementado a través de uno de los modelos pedagógicos más en auge en la investigación actual. Igualmente el artículo "Efecto del modelo de Educación Deportiva en la conducta prosocial en escolares de Educación Primaria" (Rivera et al., 2020) trata de profundizar en algunas consecuencias que puede generar el Modelo de Educación Deportiva, en este caso abordando las conductas prosociales en niños

En la línea de investigación vinculada con la intervención docente y el desarrollo de variables motivacionales 


\section{ed i toria l}

implicadas en la docencia de la Educación Física encontramos el estudio "Predicción del compromiso en Educación Física desde la teoría de la autodeterminación: análisis de invarianza según el nivel de actividad física", desarrollado por Coterón et al. (2020) y que plantea cómo las distintas relaciones entre el compromiso en Educación Física y las necesidades psicológicas básicas y la motivación intrínseca se ven afectados por el nivel de práctica de AF de los sujetos. En esta línea, el estudio "Metodologías de enseñanza-aprendizaje y su relación con la motivación e implicación del alumnado en las clases de educación física" desarrollado por Leo et al. (2020) trata de evaluar cómo distintas metodologías de enseñanza aprendizaje (i.e., constructiva, productiva, reproductiva o de acción libre) influyen en la motivación e implicación del alumnado en las clases de Educación Física. El estudio de Férriz et al. (2020), denominado "Agentes sociales de la comunidad educativa, satisfacción de novedad y actividad física", también muestra cómo la percepción de los estudiantes de un apoyo a la autonomía para adoptar un estilo de vida saludable por distintos agentes (i.e., tutor, padres, compañeros y docente de Educación Física) se relaciona con la práctica de AF. Por último, el estudio "Herramienta de Observación del Clima Interpersonal Motivacional (OCIM) para docentes de Educación Física" de Fierro-Suero et al. (2020) diseña y valida una herramienta que permitirá conocer el grado en que los docentes de Educación Física apoyan o controlan las necesidades psicológicas básicas, incluyendo también la necesidad de novedad.

Por último, con relación a la línea de investigación focalizada en el desarrollo de intervenciones desde los centros educativos o desde el área de Educación Física para el desarrollo de hábitos saludables encontramos un primer trabajo titulado "Diseño y validación de una escala para medir en profesores de Educación Física el uso responsable de las pruebas de condición física (FITPET)", de Blanco-Luengo et al. (2020), que diseña y valida una escala que permitirá evaluar el grado de uso responsable de este tipo de pruebas en contexto educativo. Por otro lado, el trabajo "Aplicación móvil Endomondo para promocionar la actividad física en estudiantes de Educación Secundaria" de Gil-Espinosa et al. (2020) presenta una experiencia didáctica, y sus resultados positivos, basada en desafíos colectivos e individuales que se gestionaban a través de esta conocida aplicación móvil. El estudio "Evaluación de la capacidad cardiorrespiratoria en estudiantes entre 12 y 19 años de Cádiz (España): desarrollo de una calculadora para estimar el esfuerzo y salud cardiovascular" de Lamoneda et al. (2020), analiza por un lado la capacidad cardiorrespiratoria en un grupo de adolescentes $y$, por otro lado, aporta una calculadora de rendimiento y esfuerzo que guíe la evaluación formativa en este contexto. Otro de los estudios de este bloque, elaborado por GómezGonzález et al. (2020) y titulado "Niveles de actividad física y sedentarismo de los adolescentes en recreos naturales vs. tradicionales: un estudio piloto", plantea las posibles diferencias de los recreos naturales y cómo pueden contribuir a aumentar los niveles de AF en la etapa adolescente, donde se construyen las identidades activas. Por último, el trabajo "Orientaciones para la comunidad científica sobre el diseño, implementación y evaluación de intervenciones escolares sobre promoción de comportamientos saludables", desarrollado por Sevil-Serrano et al. (2020), ofrece un total de ocho orientaciones metodológicas avaladas por la literatura científica para mejorar la implementación de intervenciones y que de esta forma se pueda aumentar la efectividad de las mismas.

Por último, como editores del monográfico no solo queremos agradecer a los autores de los manuscritos su interés y esfuerzo por desarrollar investigación de calidad, sino que también queremos agradecer a todos los miembros de la Red internacional de investigación en Educación Física y Promoción de Hábitos Saludables su implicación durante estos dos años y su participación activa para el desarrollo de este monográfico. 


\section{Referencias}

Beltrán-Carrillo, V. J., \& Devís-Devís, J. (2019). El pensamiento del alumnado inactivo sobre sus experiencias negativas en educación física: los discursos del rendimiento, salutismo y masculinidad hegemónica. RlCYDE. Revista Internacional de Ciencias del Deporte, 55(15), 20-34 doi:10.5232/ricyde.

Blanco-Luengo, D., Nuviala, A., Izquierdo-Gómez, R., \& Grao-Cruces, A (2020). Diseño y validación de una escala para medir en profesores de Educación Física el uso responsable de las pruebas de condición física (FITPET). Cultura, Ciencia y Deporte, 46(15), 551-560.

Borde, R., Smith, J. J., Sutherland, R., Nathan, N., \& Lubans, D. R. (2017). Methodological considerations and impact of school-based interventions on objectively measured physical activity in adolescents: a systematic review and meta-analysis. Obesity Reviews, 18(4), 476-490. doi:10.1111/obr.12517

Carriedo, A., Méndez-Giménez, A., Fernández-Rio, J., \& Cecchini, José A (2020). Enseñanza y aplicación de los test de condición física en Educación Secundaria: Una propuesta basada en el Modelo de Educación Deportiva. Cultura, Ciencia y Deporte, 46(15), 529-540.

Cassar, S., Salmon, J., Timperio, A., Naylor, P. J., van Nassau, F., Ayala, A M. C., \& Koorts, H. (2019). Adoption, implementation and sustainability of school-based physical activity and sedentary behaviour interventions in real-world settings: a systematic review. International Journal of Behavioral Nutrition and Physical Activity, 16(1), 120. doi:10.1186/s12966019-0876-4.

Coterón, J., Franco, E., \& Almena, A. (2020). Predicción del compromiso en Educación Física desde la teoría de la autodeterminación: análisis de invarianza según el nivel de actividad física. Cultura, Ciencia y Deporte, 46(15), 485-494.

Ferriz, R. González-Cutre, D., \& Balaguer-Giménez, J. (2020). Agentes sociales de la comunidad educativa, satisfacción de novedad y actividad física. Cultura, Ciencia y Deporte, 46(15), 519-528

Fierro-Suero, S., Almagro, B. J., Castillo, I., \& Sáenz-López, P. (2020). Herramienta de Observación del Clima Interpersonal Motivacional (OCIM) para docentes de Educación Física. Cultura, Ciencia y Deporte, 46(15), 575-596.

Gakidou, E., Afshin, A., Abajobir, A. A., Abate, K. H., Abbafati, C., Abbas, K. M., ... Abu-Raddad, L. J. (2017). Global, regional, and national comparative risk assessment of 84 behavioural, environmental and occupational, and metabolic risks or clusters of risks, 1990-2016: a systematic analysis for the Global Burden of Disease Study 2016. Lancet, 390(10100), 1345 1422. doi:10.1016/S0140-6736(17)32366-8.

Gasol Foundation (2019). Informe estudio PASOS 2019. Barcelona: Gaso Foundation.

Gil-Espinosa, F. J., Merino-Marbán, R. \& Mayorga-Vega, D. (2020). Aplicación móvil Endomondo para promocionar la actividad física en estudiantes de educación secundaria. Cultura, Ciencia y Deporte, 46(15), 465-473

Gómez-González, L., Valencia-Peris, A., Lizandra, J., \& Peiró-Velert, C. (2020). Niveles de actividad física y sedentarismo de los adolescentes en recreos naturales vs. tradicionales: un estudio piloto. Cultura, Ciencia y Deporte, 46(15), 475-484.

Guthold, R., Stevens, G. A., Riley, L. M., \& Bull, F. C. (2019). Global trends in insufficient physical activity among adolescents: a pooled analysis of 298 population-based surveys with 1.6 million participants. The Lancet Child \& Adolescent Health, 4(1), 23-35. doi:10.1530/ey.17.13.12

Hagger, M. S., \& Chatzisarantis, N. L. (2012). Transferring motivation from educational to extramural contexts: A review of the trans-contextual model. European Journal of Psychology of Education, 27(2), 195-212. doi:10.1007/s10212-011-0082-5
Hollis, J. L., Sutherland, R., Williams, A. J., Campbell, E., Nathan, N., Wolfenden, L., ... Wiggers, J. (2017). A systematic review and meta-analysis of moderate-to-vigorous physical activity levels in secondary school physical education lessons. International Journal of Behavioral Nutrition and Physical Activity, 14, 52. doi:10.1186/s12966-017-0504-0.

Ladwig, M. A., Vazou, S., \& Ekkekakis, P. (2018). "My best memory is when I was done with it": PE memories are associated with adult sedentary behavior. Translational Journal of the American College of Sports Medicine, 3(16), 119-129. doi: 10.1249/TJX.0000000000000067

Lamoneda, J., Huertas, F. J., y Cadenas, C. (2020). Evaluación de la capacidad cardiorrespiratoria en estudiantes entre 12 y 19 años de Cádiz (España): desarrollo de una calculadora para estimar el esfuerzo y salud cardiovascular. Cultura, Ciencia y Deporte, 46(15), 541-549.

Leo, F. M., López-Gajardo, M. A., Gómez-Holgado, J. M., Ponce-Bordón, J. C., \& Pulido, J. J. (2020). Metodologías de enseñanza-aprendizaje y su relación con la motivación e implicación del alumnado en las clases de Educación Física. Cultura, Ciencia y Deporte, 46(15), 495-506.

Love, R. E., Adams, J., \& van Sluijs, E. M. F. (2017). Equity effects of children's physical activity interventions: a systematic scoping review. International Journal of Behavioral Nutrition and Physical Activity, 14, 134. doi:10.1186/s12966-017-0586-8

Luque, A., \& Del Villar, F. (2019). Mujer joven y actividad física. Madrid Fundación Mapfre.

Marques, A., Loureiro, N., Avelar-Rosa, B., Naia, A., \& de Matos, M. G. (2018). Adolescents' healthy lifestyle. Jornal de Pediatria, 96(2), $217-$ 224 doi: 10.1016/j.jped.2018.09.002

Moreno, C., Ramos, P., Rivera, F., Sanchez-Queija, I., Jiménez-Iglesias, A., ... Leal-López, E. (2019). La adolescencia en España: salud, bienestar, familia, vida académica y social. Resultados del Estudio HBSC 2018. Ministerio de Sanidad, Consumo y Bienestar Social. Madrid.

Nguyen, S., Häcker, A. L., Henderson, M., Barnett, T., Mathieu, M. E., Pagani, L., \& Bigras, J. L. (2016). Physical activity programs with postintervention follow-up in children: a comprehensive review according to categories of intervention. International Journal of Environmental Research and Public Health, 13(7), 664. doi:10.3390/ijerph13070664

Rivera, M.C., Gutiérrez, D., Segovia, Y., \& Valenciano, J. (2020). Efecto del modelo de Educación Deportiva en la conducta prosocial en escolares de Educación Primaria. Cultura, Ciencia y Deporte, 46(15), 561-574.

Sallis, J. F. (2018). Needs and challenges related to multilevel interventions: physical activity examples. Health Education \& Behavior, 45(5), 661 667. doi:10.1177/1090198118796458

Sevil-Serrano, J., Abós, A., Aibar, A., Simón-Montañés, L., \& García-González, L. (2020). Orientaciones para la comunidad científica sobre el diseño, implementación y evaluación de intervenciones escolares sobre promoción de comportamientos saludables. Cultura, Ciencia y Deporte, 46(15), 507-517.

Slingerland, M., \& Borghouts, L. (2011). Direct and indirect influence of physical education-based interventions on physical activity: a review. Journal of Physical Activity and Health, 8(6), 866-878. doi:10. 1123/jpah.8.6.866

Taylor, I. M., Ntoumanis, N., \& Smith, B. (2009). The social context as a determinant of teacher motivational strategies in physical education. Psychology of Sport and Exercise, 10(2), 235-243. doi:10.1016/j. psychsport.2008.09.002

Vasconcellos, D., Parker, P. D., Hilland, T., Cinelli, R., Owen, K. B., Kapsal, N., ... Lonsdale, C. (2020). Self-determination theory applied to physical education: A systematic review and meta-analysis. Journal of Educational Psychology, 112(7), 1444-1469. doi:10.1037/edu0000420

Whitehead, M. (2010). Physical literacy: Throughout the lifecourse. Reino Unido: Routledge. 